\title{
Effect of semen collection methods on the quality of pre- and post-thawed Bali cattle (Bos javanicus) spermatozoa.
}

\begin{abstract}
This study was conducted to evaluate the response of Bali bulls (Bos javanicus) to different semen collection methods and their effects on fresh and post-thawed semen quality. The collection methods employed were electro-ejaculation (EE), transrectal massage (RM) and $\mathrm{RM}$ followed by EE (RM + EE). A total of 25 untrained Bali bulls (age between 2 and 4 years old) were subjected to the different semen collection methods. Fresh semen samples from all the 25 bulls were evaluated for volume, $\mathrm{pH}$, general motility, live/dead ratio and abnormality using the conventional method. For fresh and frozen samples collected by EE and RM from 10 bulls, computer-assisted semen analysis system was used for precise quantitative measurement of motility, velocity and forward progression. Accucell photometer was used to measure sperm concentration in all samples, regardless fresh and frozen. Semen samples were obtained $100 \%$ of the attempts using EE, $84 \%$ using RM and $96 \%$ using RM + EE. There were no differences among the collection methods for fresh semen quality characteristics, including motility, morphology and viability, but $\mathrm{pH}$ and volume were higher for EE than RM and RM + EE. Higher sperm concentration was observed in semen collected by RM than the other two methods. Different age groups (2-3 and >3-4 years old) of the bulls did not show significant differences in volume, $\mathrm{pH}$, sperm concentration, percentages in motility, live/dead ratio and normal sperm morphology. The quality of semen for general and progressive motility, VAP, VSL and VCL and acrosomal integrity after thawing was higher for RM than EE. In conclusion, Bali bulls appeared to respond best to EE and the combination of RM + EE than RM, as a method of semen collection, with a shorter time of stimulation required. Differences in age of the Bali bulls did not affect the semen quality.
\end{abstract}

Keyword: Bali cattle; Electroejaculation; Rectal massaging; Semen quality; Cryopreservation. 